\title{
EVOLUTION AND DEVELOPMENT IN THE ORIGIN OF MAJOR GROUPS OF VASCULAR PLANTS
}

STEIN, William E., Center for Evolution and Paleoenvironment, State University of New York, Binghamton, N.Y. 13902-6000. U.S.A.

Among multicellular organisms, the fossil record of vascular plants is, perhaps, uniquely informative about pattern and process leading to the establishment of major groups. Although classifications above the family level remain highly debatable, especially with regard to level, important insight has been gained into external morphology and internal anatomy of the earliest members of the clade, as well as into general patterns of diversification and change in form through time. Despite these advantages, cladistic methods have brought into sharp focus significant problems with important characters at high taxonomic levels. Putative synapomorphies used to diagnose major groups often have conflicting phylogenetic implications which, according to the cladistic paradigm, must be interpreted as homoplasy. From a biological standpoint, however, unrecognized instances of homoplasy in the character set often seem unlikely due to the complexity of the features involved, and the lack of plausible mechanisms producing multiple parallelisms or reversals by means of "known" processes such as adaptation, homeosis, heterochrony, or functional constraint. Added to this are conceptual and practical problems concerning morphological gaps (and what might bridge them) between recognized groups. One of the most interesting challenges to current approaches are enigmatic fossils early in the history of apparent clades exhibiting unprecedented variability in expression of supposedly diagnostic features. As a result, terminology originally developed to circumscribe alternative morphological states within clades completely breaks down. Perhaps, what's needed here is a more dynamic model of evolutionary change linking hypotheses of synapomorphy with specific changes in the structuring "rules" (or capacities) of development.

Among characters used to diagnose major groups of vascular plants, differences in stelar architecture remain among the most important. Comparative evidence suggests that early vascular plants have essentially the same relatively well-understood developmental mechanisms as living plants; vascular tissues differentiate according to hormone (primarily auxin) gradient signals, that are the more-or-less the passive consequence of shoot geometry during growth. Because early plants have an extremely simple organography (dichotomizing stems with no leaves), an unparalleled opportunity exists to understand historical changes leading to establishment of major stelar morphs in the fossil record terms of changed dynamics of continuous serial development at the shoot apex. A computer simulation of vascular tissue patterning under hormone influence will be presented, and suggestions offered regarding the relative plausibility of alternate routes of evolutionary change between major groups. 